\title{
Performance of the Upper Limb Module for DMD 1.2 Functional Test
}

National Cancer Institute

\section{Source}

National Cancer Institute. Performance of the Upper Limb Module for DMD 1.2

Functional Test. NCl Thesaurus. Code C138334.

A 22-item assessment scale developed by an international clinical outcomes group in 2011 to measure motor performance of the upper limb in patients with Duchenne muscular dystrophy (DMD). Functional tasks are subdivided into three dimensions that reflect disease progression from proximal to distal weakness, and from ambulatory to non-ambulatory. 